\title{
BMJ Open Specific growth rates calculated from CTs in patients with head and neck squamous cell carcinoma: a retrospective study performed in Austria
}

\author{
Daniel Dejaco, ${ }^{\oplus 1}$ Teresa Steinbichler, ${ }^{1}$ Volker Hans Schartinger, ${ }^{1}$ Natalie Fischer, ${ }^{1}$ \\ Maria Anegg, ${ }^{1}$ Joszef Dudas, ${ }^{1}$ Andrea Posch, ${ }^{2}$ Gerlig Widmann, ${ }^{3}$ \\ Herbert Riechelmann ${ }^{1}$
}

To cite: Dejaco D, Steinbichler T, Schartinger VH, et al. Specific growth rates calculated from CTs in patients with head and neck squamous cell carcinoma: a retrospective study performed in Austria. BMJ Open 2019:9:e025359. doi:10.1136/ bmjopen-2018-025359

- Prepublication history for this paper is available online. To view these files, please visit the journal online (http://dx.doi org/10.1136/bmjopen-2018025359).

This work has been previously presented at the 62nd anual conference of the Austrian EarNose-Throat society in october 2018 in Bregenz, Vorarlberg, Austria.

Received 12 July 2018 Revised 19 December 2018 Accepted 27 December 2018

Check for updates

(c) Author(s) (or their employer(s)) 2019. Re-use permitted under CC BY-NC. No commercial re-use. See rights and permissions. Published by BMJ.

${ }^{1}$ Department of

Otorhinolaryngology-Head and Neck Surgery, Medical University of Innsbruck, Innsbruck, Austria ${ }^{2}$ Department of Radiation Oncology, Medical University of Innsbruck, Innsbruck, Austria

${ }^{3}$ Medical University of Innsbruck, Department of Radiology, Innsbruck, Austria

Correspondence to Dr Daniel Dejaco; daniel.dejaco@i-med.ac.at

\section{ABSTRACT}

Objective To provide data on specific growth rates (SGRs) of primary tumours (PT-SGR) and largest pathological cervical lymph nodes (LN-SGR) for head and neck squamous cell carcinoma (HNSCC). To explore PT-SGR's and LN-SGR's correlation with selected biomarkers epidermal growth factor receptor (EGFR), Ki67 and CD44. Design and setting Retrospective study performed at a tertiary oncological referral centre in Innsbruck, Austria. Participants Adult patients with incident HNSCC treated with primary radiotherapy (RT) or radiochemotherapy (RCT).

Outcome measures Volumes of the primary tumour (PT-volume) and largest pathological cervical lymph node (LN-volume) were measured in CT scans obtained at time of diagnosis and subsequent planning CTs immediately prior to RT or RCT. SGRs were calculated assuming an exponential growth function. PT-SGR's and LN-SGR's correlation with EGFR, Ki67 and CD44 were explored. Results In 123 patients, mean interval between diagnostic and planning CT was $29 \pm 21$ days. PT-SGR was $1.8 \pm 1.8 \%(m e a n \pm S D)$ per day and was positively correlated with EGFR, Ki67 and CD44 expression $(\mathrm{p}=0.02$; $\mathrm{p}=0.02 ; \mathrm{p}=0.03$ ). $\mathrm{LN}-\mathrm{SGR}$ was $1.7 \pm 2.0 \%$ per day and increased with larger initial LN-volume, was lower in laryngeal cancer $(\mathrm{p}=0.003)$ and slowed down with time. LN-SGR was not correlated with EGFR, Ki67 or CD44 expression in primary tumours $(p>0.12)$. New cartilage or bone infiltration occurred in 10 patients and new central lymph node necrosis in 8 patients.

Conclusions HNSCCs are fast-growing tumours for which treatment must not be delayed. Clinical tumour growth rates are influences by EGFR, KI67 and CD44 expression.

\section{INTRODUCTION}

In patients with head and neck squamous cell carcinoma (HNSCC), the median treatment waiting time in the USA almost doubled from 19 to 30 days between 1998 and 2011. In a recent cancer registry-based study, Murphy and coauthors analysed almost 275000 patients with HNSCC of the most common cancer sites. The authors observed
Strengths and limitations of this study

- In patients with incident head and neck squamous cell carcinoma, specific growth rates (SGRs) for primary tumours (PTs) and largest pathological cervical lymph nodes (LNs) were retrospectively calculated.

- SGR in percentage growth per day was calculated from two CT scans obtained at time of diagnosis and subsequent planning CTs immediately prior to radiochemotherapy as previously described (SGR $=\ln (1 \mathrm{st}$ volume $\times 2 \mathrm{nd}$ volume $\left.) /\left(\mathrm{t}_{2}-\mathrm{t}_{1}\right)\right)$.

- Volumes in millilitres for PT and LN were calculated from maximum orthogonal diameters in all three planes applying an ellipsoid formula as previously described (volume $\left.=\left(\pi^{*}\left(x^{*} y^{\star} z / 1000\right)\right) / 6\right)$.

- To explore the impact of SGR of PT and LN on overall survival, Kaplan-Meier and Cox regression models were used; to explore the correlation of SGR with epidermal growth factor receptor, Ki67 and CD44, Jonckheere-Terpstra tests were used.

- Limitations include retrospective study design, small number of patients, small interval of observation, and lack of more modern imaging and segmentation techniques.

an independent effect of increased treatment waiting time on overall survival $(\mathrm{OS})^{1}$ and calculated 46 to 52 days as threshold for decreased OS. ${ }^{2}$ Similar observations were reported for oral cancer in a recent review including 18 studies. ${ }^{3}$

A likely reason for the association of treatment waiting time and decreased OS is meantime tumour growth. Mathematical models to approximate tumour growth from imaging data are available since the 1960 s. $^{4}$ Originally, direct curve fitting to calculate tumour volume doubling time (DT) was the standard method to assess tumour growth. ${ }^{4}$ Recently, calculation of specific growth rate (SGR), defined as relative volume increase per unit of time, was proposed instead. It was reported 
more reliable for short time intervals and minor tumour volume differences. ${ }^{5}$

Data on SGR of HNSCC are limited. ${ }^{67}$ A median SGR for primary tumours (PT-SGR) of $0.74 \%$ per day in patients with oropharyngeal HNSCC waiting for radiochemotherapy (RCT) was reported by Murphy and colleagues. The authors assessed the PT-SGR in 85 patients between diagnostic CTs and planning CTs and concluded that rapid PT-SGR may predict treatment failure in these patients. ${ }^{6}$ Van Bockel and coauthors reported a significant association between high PT-SGR and decreased OS ( $p=0.013)$ in 131 patients with laryngeal HNSCC. ${ }^{7}$

In this retrospective study, we calculated $\mathrm{SGR}^{5}$ of the primary tumour (PT-SGR) and largest pathological cervical lymph node (LN-SGR) of patients with incident HNSCC from CTs obtained at diagnosis and from planning CTs obtained directly before radiotherapy (RT) / RCT. We investigated the influence of various factors including several biomarkers on PT-SGR and LN-SGR, which were previously observed to be associated with tumour proliferation. ${ }^{8-12}$ We were further interested in the influence of SGR on OS and on the development of new lymph node necrosis and bone or cartilage infiltration.

\section{MATERIALS AND METHODS}

\section{Tumour registry population}

Patients referred to the Department of Otorhinolaryngology-Head and Neck Surgery, Medical University of Innsbruck, Austria, between 2008 and 2016 with incident histologically confirmed HNSCG were recorded in the clinical tumour registry. Disease was staged according to the seventh edition of the Union internationale contre le cancer Tumor-Node-Metastasis TNM staging system ${ }^{13}$ by an interdisciplinary tumour board. Inclusion criteria comprised histologically proven incident HNSCC at any site of the head and neck region including cancer of unknown primary (CUP), any UICC stage, RT or RCT as primary treatment, and availability of both a diagnostic CT and a planning CT.

\section{Diagnostic CT and planning CT}

At the time of clinical diagnosis, diagnostic CT was performed following the standardised CT head and neck imaging protocols at the Department of Radiology, Medical University of Innsbruck. A GE Medical Systems Light Speed VCT or Light Speed 16 CT scanner (GE Medical, Vienna, Austria) was used. The scan area ranged from the frontal sinus to the upper mediastinum with a resolution of $512 \times 512$ pixels. Slices were calculated from raw data with $2 \mathrm{~mm}$ thickness, collimation of $24 \times 1.2 \mathrm{~mm}$ and 0.45 pitch. Additional sagittal and coronal images were reconstructed. As contrast medium, Jopamiro 370 (Bracco Austria GmbH, Vienna, Austria) was administered intravenously adjusted to the patient's body weight. Planning CT scans were later performed at the Department of Radiation Oncology, Medical University of Innsbruck. Imaging protocols were followed as described

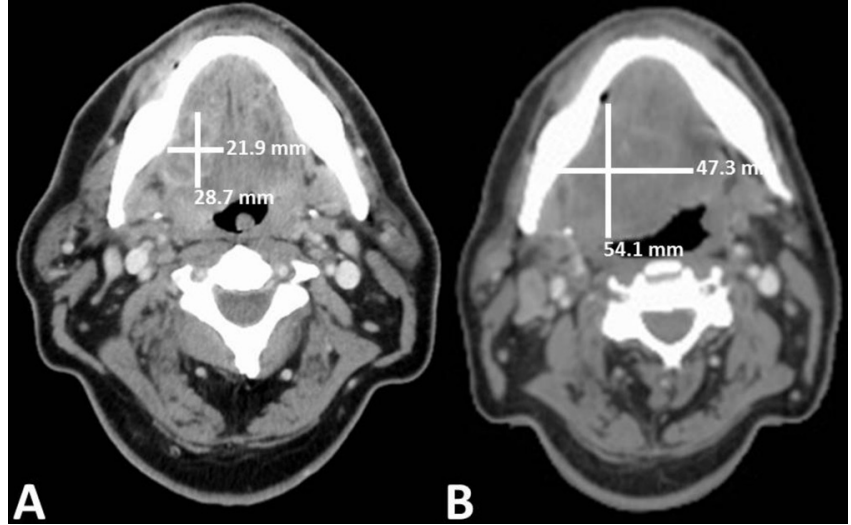

Figure 1 Tumour growth assessment using maximal orthogonal tumour diameters from axial contrast-enhanced diagnostic CT scans $(A)$ and subsequent planning CT scans (B). Axial contrast-enhanced diagnostic CT scan (A) and subsequent planning CT scan (B) 46 days later of a cT4a cN2b cM0 squamous cell carcinoma of the oral cavity. The maximum anterior-posterior and mediolateral tumour diameters (white lines) were measured from axial scans; the craniocaudal tumour diameters were measured from corresponding coronal scans (not depicted). The tumour volume was assessed as previously described using an ellipsoid formula. ${ }^{15}$ Volume of the primary tumour (PT-volume) from diagnostic CT was $14.8 \mathrm{~mL}$, PT-volume from planning CT was $51.0 \mathrm{~mL}$ translating to a specific growth rate of primary tumours of $2.8 \% /$ day.

above with the same CT scanners, contrast medium, scanning areas, resolutions and calculation protocols. Images from both CT scans were exported in Digital Imaging and Communications in Medicine format using IMPAX EE (Agfa HealthCare, Bonn, Germany) Picture Archiving and Communication System (Cerner, Kansas City, Missouri, USA). LN with a minimal axial diameter $>10 \mathrm{~mm}$, a central necrosis $>3 \mathrm{~mm}$ or if present in neck levels close to the primary tumour in groups of $>3$ were classified pathological. ${ }^{14}$

\section{Volume approximation, SGR and tumour volume DT}

Volumes were calculated as previously described. ${ }^{15}$ In short, maximum orthogonal diameters $(\mathrm{mm})$ were measured for the primary tumour (PT) and the largest pathological cervical lymph (LN) in all three planes in axial and coronal sections (figure 1). Volumes (mL) were approximated employing an ellipsoid formula (volume $\left.=\left(\Phi^{*}\left(\mathrm{x}^{*} \mathrm{y}^{*} \mathrm{z} / 1000\right)\right) / 6\right)$. The largest cervical LN instead of all pathological cervical lymph nodes was considered sufficient for evaluation based on a high correlation previously observed. ${ }^{15}$ Central lymph node necrosis and/or cartilage or bone infiltration of the primary tumour was recorded for additional analysis.

SGRs were assumed to be exponential and defined as the relative volume increase given in per cent per day. For calculation of SGR, the equation described by Mehrara and coauthors was applied (SGR $=\ln (1$ st volume $\times 2$ nd volume $\left.) /\left(t_{2}-t_{1}\right)\right){ }^{5}$ For comparison with earlier studies, DTs for primary tumours (PT-DT) and for largest 
pathological cervical lymph nodes (LN-DT) were calculated as the time difference $\times \mathrm{LN}$ divided by the logarithm of the volume ratio of the two observations. ${ }^{16}$

\section{Analysis of EGFR, Ki67 and CD44 expression}

Tumour biopsies were collected in $4 \%$ buffered formalin, fixed overnight and embedded using the ethanol-isopropanol-wax quick $4 \mathrm{~mm}$ protocol of Histos 5 embedding processor (Milestone, Bergamo, Italy). Five-micrometre-thick paraffin sections were dewaxed and antigen retrieval was performed in a Discovery automated staining system (Ventana, Tucson, Arizona, USA). Primary antibodies were added to the sections by automatic dispensing either as ready-to-use, pre-diluted, stabilised solutions provided by the manufacturers: cyclin-dependent kinase inhibitor 2A (p16) INK4 (Ventana, cat. no. 6595294001), Ki67 antigen (Ki67; Linaris E059, clone MIB-1, Dossenheim, Germany), CD44 antigen (CD44; Diagnostic Biosystems/Antibodies Online, ABIN1020059, Aachen, Germany) and epidermal growth factor receptor (EGFR; Invitrogen, Vienna, Austria). Selection of these three biomarkers was based on which were previous observations about their possible association with tumour proliferation $^{8-12}$ : (1) EGFR is a cell surface receptor which promotes proliferation, invasion, angiogenesis and metastatic spread in HNSCC, if overexpressed. ${ }^{8}$ Thus, high expression of EGRF might suggest higher PT-SGR. (2) Ki67 is a nuclear protein expressed on cells in all phases of the cell cycle except in G0 phase. Thus, its expression marks the total fraction of proliferation cells in a tumour, ${ }^{9-11}$ suggestive of a possible positive correlation with PT-SGR. (3) Positive correlations between CD44 expression and advanced $\mathrm{T}$ categories were previously reported in a meta-analysis including 30 studies with 2102 patients. ${ }^{12}$ Since T category is primarily based on maximal tumour diameter, a possible positive correlation between CD44 expression and PT-SGR might be suggested. Immunohistochemical staining was completed by the Discovery automated staining system (Ventana) using universal secondary antibody solution, haematoxylin counterstaining and the DAB MAP Kit (all Ventana products) as published previously. ${ }^{17}$ All sections were stained with control mouse and rabbit immunoglobulins, using the same highest concentration as for the primary antibodies, and these controls were not reactive. ${ }^{18}$ The immunohistochemical reactions were observed independently by two blinded observers, who collected 10 representative tumour cell nests from each specimen. ${ }^{19}$ These regions were analysed on an Olympus BX50 microscope (Olympus, Tokyo, Japan) and the staining intensity and representation of tumour cell nests were scored as previously described.$^{20}$ The cut-off for p16 positivity was $70 \%$ or more positive tumour cells. ${ }^{21}$

\section{Data analysis}

Frequency data were presented in tabular form. For continuous data (volumes and specific growth rates), means and SD as well as medians and 25th (p25th) and
75 th percentiles $(\mathrm{p} 75$ th) were provided. The median follow-up time was calculated as described by Schemper and Smith. ${ }^{22}$ Logarithmic transformation was used to analyse volumetric data in regression models. Kruskal-Wallis and Jonckheere-Terpstra tests were used to evaluate the univariate influence of ordinal factors on growth rates. For survival analyses, Kaplan-Meier and Cox regression models were used. For Kaplan-Meier plots, growth rates were categorised in groups with slow, intermediate and rapid growth rates. All calculations were performed with SPSS V.23.0.

\section{Patient and public involvement}

The development of the research question was based on previous publications exploring tumour volume in HNSCC $^{15}$ and its prognostic value if treated primarily with surgery. ${ }^{23}$ Neither patients nor the public were involved in the design of the study, the recruitment of the study or the conduct of the study.

\section{RESULTS \\ Study population}

Between 2008 and 2016, 802 patients with incident HNSCC were recorded in the clinical cancer registry. Of 123 patients treated with primary RT or RCT, PT-volumes and LN-volumes were calculated from diagnostic and planning CTs. Tumour sites included oral cavity, oropharyngeal, hypopharyngeal and laryngeal HNSCC. No patients with tumours of the nasopharynx, the paranasal sinuses or salivary glands were included. In five patients with CUP syndrome, no PT-volume and in 27 patients with N0 stage neck, no LN-volume could be measured. Of the 123 patients, 32 were women. The mean \pm SD age was $63 \pm 10$ years ranging from 38 to 87 years. Median follow-up time was 45 months (95\% CI 42 to 48 months). Additional clinical data of the 123 included patients are provided in table 1 .

\section{Time interval, tumour and lymph node volumes}

Mean \pm SD time interval between diagnostic CTs and planning CTs was 29 21 days ranging from 6 to 146 days. For PT, a total of 119 volume sets from diagnostic CT and planning CT were available (figure 2). Mean PT-volume from diagnostic CTs was $16.3 \pm 20.4 \mathrm{~mL}$ ranging from 0 (CUP syndrome) to $100.8 \mathrm{~mL}$. Mean PT-volume from planning CTs was $24.5 \pm 26.8 \mathrm{~mL}$ ranging from 0 to $160.3 \mathrm{~mL}$. The mean PT-volume increase of $6.7 \pm 17.2 \mathrm{~mL}$ during the period between first and second CT scans was highly significant $(\mathrm{p}<0.001)$.

For LN, a total of 96volume sets were available (figure 2). Mean LN-volume from diagnostic CTs was $9.2 \pm 22.2 \mathrm{~mL}$ ranging from $0(\mathrm{~N} 0)$ to $156.2 \mathrm{~mL}$. Mean $\mathrm{LN}$-volume from planning $\mathrm{CTs}$ was $15.2 \pm 31.5 \mathrm{~mL}$ ranging from 0 to $233.5 \mathrm{~mL}$. The mean $\mathrm{LN}$-volume increase of $5.3 \pm 17.3 \mathrm{~mL}$ during the observation period was highly significant $(\mathrm{p}<0.001)$. Distributions of these parameters 
Table 1 Clinical data of included 123 patients with head and neck squamous cell carcinoma

\begin{tabular}{lc}
\hline & Patients (n) \\
\hline Sex & \\
\hline Male & 89 \\
\hline Female & 32 \\
\hline Age (years) & \\
\hline$\leq 50$ & 11 \\
\hline $51-60$ & 42 \\
\hline $61-70$ & 39 \\
\hline $71-80$ & 26 \\
\hline$\geq 80$ & 5 \\
\hline p16 & \\
\hline Negative & 83 \\
\hline Positive & 38 \\
\hline Tumour site & \\
\hline Oral cavity & 16 \\
\hline Oropharynx & 55 \\
\hline Hypopharynx & 24 \\
\hline Larynx & 21 \\
\hline Carcinoma of unknown primary & 5 \\
\hline Clinical UICC stage & 75 \\
\hline Stage I & \\
\hline Stage II & \\
\hline Stage III & \\
\hline Stage IVa & \\
\hline Stage IVb & \\
\hline Stage IVc & \\
\hline & \\
\hline
\end{tabular}

were right skewed. Medians and quartiles for PT-volume and LN-volume are provided in table 2.

\section{SGRs and tumour DTs}

Mean \pm SD PT-SGR was $1.8 \pm 1.8 \% /$ day ranging from $-2.6 \% /$ day (volume decrease) to $8.6 \% /$ day. Mean
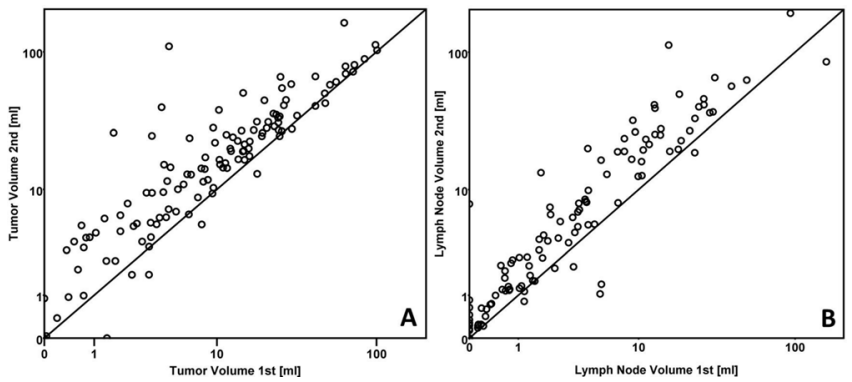

Figure 2 Correlation of primary tumour volume (A) and largest pathological lymph node volume (B) measured from diagnostic CT scans and planning CT scans. Scattergram of primary tumour volumes $(A)$ and largest pathological lymph node volume (B) measured from diagnostic CT scans ( $\mathrm{x}$-axis) and planning CT scans (y-axis). Both axes are on log scale. The diagonal line represents the line of identity. Dots above this line indicate volume increases.
LN-SGR was $1.7 \pm 2.0 \%$ / day ranging from $-1.5 \%$ to $11.0 \%$ / day. PT-SGR and LN-SGR were right-skewed. For medians and quartiles see table 2. Mean PT-SGR of tumours of the oral cavity, oropharynx, hypopharynx and larynx were $3.1 \pm 1.5 \% /$ day, $1.8 \pm 1.7 \% /$ day, $2.0 \pm 2.0 \% /$ day and $1.5 \pm 2.1 \% /$ day, respectively. Mean LN-SGR for the primary tumour sites oral cavity, oropharynx, hypopharynx and larynx were $3.4 \pm 3.9 \% /$ day, $1.9 \pm 2.2 \% /$ day, $1.9 \pm 1.4 \%$ / day and $0.8 \pm 1.1 \% /$ day, respectively. For tumour sites, medians and quartiles are provided in table 3 .

Median PT-DT was 43 days. The 25 th percentile was 24 and the 75 th percentile 85 days. Median LN-DT was 41 days. The 25 th percentile was 25 and the 75 th percentile 80 days (table 2). PT-DT and LN-DT were considerably right skewed.

\section{Factors influencing SGRs}

PT-SGR was independent of the initial PT-volume in diagnostic CT $(p=0.19)$. Also, PT-SGR did not depend on the interval between first and second CT $(p=0.14)$. Moreover, tumour site had no significant impact on SGR ( $\mathrm{p}=0.58$; table 3). Interestingly, PT-SGR positively correlated with the expression of EGFR, Ki67 and CD44 ( $p=0.02$, $p=0.02$ and $p=0.03$, respectively; figure 3 ). The expression of 16 had no influence on PT-SGR ( $p=0.21)$.

In contrast, LN-SGR depended on the initial LN-volume measured in diagnostic CT $(\mathrm{p}=0.003)$ with higher LN-SGRs in lymph nodes with larger LN-volumes in the first CT. Also, the interval between the diagnostic CT and planning CT significantly influenced LN-SGR ( $\mathrm{p}=0.003)$. The longer the interval, the lower the observed LN-SGR. Moreover, LN-SGR was significantly influenced by tumour site $(p=0.032$; table 3$)$ with smallest growth rates in lymph nodes from laryngeal HNSCC $(p=0.003)$. In contrast to PT-SGR, neither EGFR, Ki67, CD44 nor p16 expression in PTs significantly correlated with LN-SGR $(p=0.12$, $\mathrm{p}=0.31, \mathrm{p}=0.75$ and $\mathrm{p}=0.81$, respectively).

\section{SGR and OS}

PT-SGR nearly missed significant impact on OS when used as a single covariate in a Cox regression model (log-rank $\mathrm{p}=0.054)$. For Kaplan-Meier analyses, PT-SGR were categorised in three groups: slow $(<0.3 \% /$ day; $n=22)$, intermediate $(0.3 \%$ to $\leq 3 \% /$ day; $\mathrm{n}=73)$ and rapid $(>3 \% /$ day; $\mathrm{n}=26$ ). Survival curves of these three SGR groups did not differ significantly (log-rank $\mathrm{p}=0.45$; figure 4 ). Likewise, LN-SGR had no significant impact on OS as a covariate in Cox regression $(\log$-rank $\mathrm{p}=0.83$ ) nor in Kaplan-Meier analyses (log-rank $\mathrm{p}=0.97$; data not shown).

\section{Cartilage or bone infiltration and central lymph node necrosis}

Cartilage or bone infiltration was observed in diagnostic CTs of 40/123 (33\%) patients and in planning CTs of $50 / 123(41 \%)$ patients. Thus, during the median 24-day interval between the two CTs, new cartilage or bone infiltration occurred in 10 patients, which was not influenced by PT-SGR $(p=0.918)$. However, cartilage or bone infiltration had a significant negative impact on survival 
Table 2 Volumes of primary tumours and largest pathological cervical lymph nodes in diagnostic and planning CTs in 123 patients with head and neck squamous cell carcinoma

\begin{tabular}{|c|c|c|c|c|}
\hline & 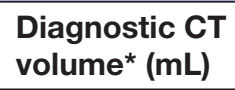 & $\begin{array}{l}\text { Planning CT } \\
\text { volume }^{*}(\mathrm{~mL})\end{array}$ & SGR† (\%/day) & DT‡ \\
\hline Primary tumour & $9.4(3.3 ; 21.4)$ & $16.7(5.4 ; 31.6)$ & $1.4(0.6 ; 2.7)$ & $43(24 ; 85)$ \\
\hline
\end{tabular}

Median time interval between 1st and 2nd CT scans was 24 days. Specific growth rates were calculated as suggested by Mehrara and coauthors, ${ }^{5}$ tumour doubling times were calculated as proposed by Schwartz. ${ }^{16}$

${ }^{*}$ Median (25th and 75th percentiles).

†Specific growth rate (median, 25th and 75th percentiles) per cent per day.

$\ddagger$ Tumour doubling time in days (median, 25th and 75th percentiles).

$(\mathrm{p}=0.003)$ and was significantly more frequent in $\mathrm{p} 16-\mathrm{neg}$ ative tumours $(\mathrm{p}=0.02)$.

Lymph node necrosis was observed in diagnostic CTs of 74/97 (76\%) patients. LN-SGR was significantly higher in patients with lymph node necrosis $(\mathrm{p}<0.001)$, was associated with poorer survival $(\mathrm{p}=0.03)$ and did not depend on p16 status. Lymph node necrosis was observed in planning CTs of $82 / 97$ (85\%) patients. Thus, during the median 24-day interval between the two CTs, new lymph node necrosis occurred in eight patients, which was not influenced by LN-SGR $(p=0.818)$. Central lymph node necrosis had a significant negative impact on survival $(\mathrm{p}<0.05)$.

\section{DISCUSSION}

Long waiting times for treatment of head and neck cancer result in decreased OS. ${ }^{1-3}$ One reason might be meantime tumour growth. We examined tumour growth during the time between the initial diagnostic CT scan and the planning CT for RT or RCT in 123 patients with incident HNSCC. The majority of the patients suffered from incident stage IVa oropharyngeal HNSCC.

The mean interval between first and second CT was 29 days ranging from 6 to 146 days. In this time, diagnostic work-up, interdisciplinary tumour board presentation and pretreatment procedures including dental treatments and application of percutaneous gastrostomies were carried out. PT-volume and LN-volume in

Table 3 Specific growth rates (SGRs) for primary tumours (PT) and largest pathological cervical lymph nodes (LN) for common tumour sites of 123 patients with incident head and neck squamous cell carcinoma

\begin{tabular}{llll}
\hline Tumour site & $\mathbf{n}$ & PT-SGR $^{*}$ & LN-SGR $^{*}$ \\
\hline Oral cavity & 15 & $2.4(1.0 ; 3.9)$ & $0.8(0.0 ; 1.5)$ \\
Oropharynx & 55 & $1.4(0.7 ; 2.6)$ & $2.5(0.4 ; 2.6)$ \\
Hypopharynx & 25 & $1.7(0.6 ; 2.7)$ & $2.1(0.6 ; 2.9)$ \\
Larynx & 21 & $1.0(0.3 ; 3.1)$ & $0.8(0.0 ; 1.4)$ \\
$\begin{array}{l}\text { Carcinoma of } \\
\text { unknown primary }\end{array}$ & 5 & $0.0(0.0 ; 0.0)$ & $1.4(1.1 ; 5.2)$ \\
\hline
\end{tabular}

${ }^{*}$ Median (25th and 75th percentiles). diagnostic and planning CTs were assessed using identical protocols. PT-SGR and LN-SGR were calculated as proposed by Mehrara and coauthors. ${ }^{5}$ This mean interval is comparable with previous reports. ${ }^{1}$ Moreover, it is considerably shorter than intervals of 46 to 52 days, which were reported as threshold for decreased OS. ${ }^{2}$ From a clinical perspective, this is considered positive. However, in terms of exploring SGRs in advanced-stage HNSCC, this interval may not be sufficient to accurately determine great changes in PT-volume or LN-volume.

Due to the skewness of volumetric data, the median was considered an appropriate measure of central tendency, but the mean is also provided for comparison with previous publications. Mean PT-volume in diagnostic CTs

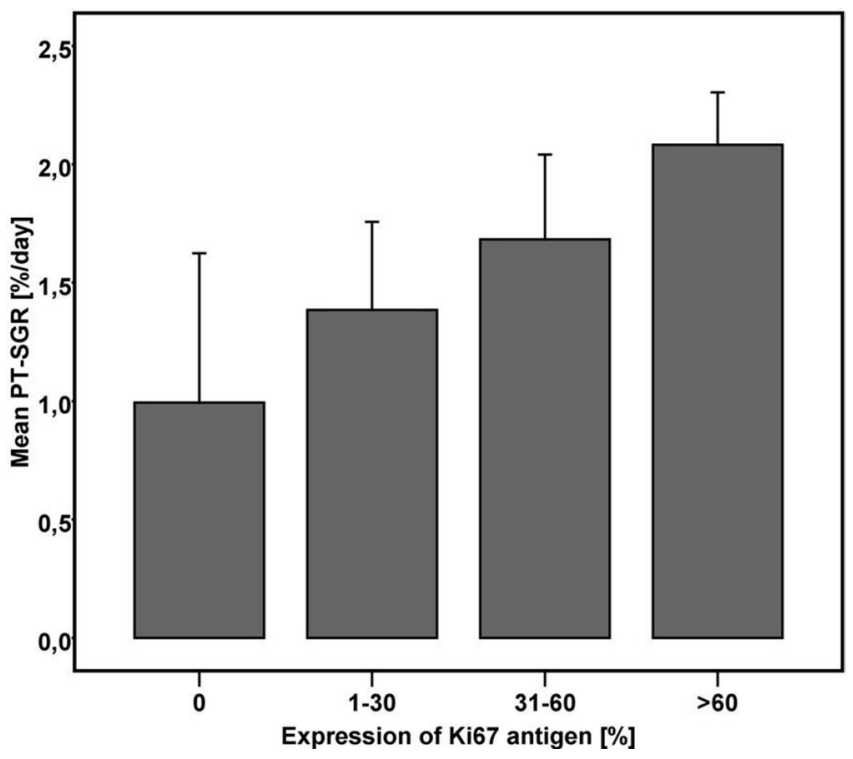

Figure 3 Specific growth rates of the primary tumours and percentage of Ki67-positive cells. Percentage of Ki67positive immunohistochemistry in tumour samples of patients with head and neck squamous cell carcinoma (HNSCC) and according primary tumour specific growth rates. The percentage of Ki67-positive cells was grouped in 0\%, 1\% to $30 \%, 31 \%$ to $60 \%$ and more than $60 \%$ of cancer cells (x-axis). Mean specific growth rates of HNSCC primary tumour (y-axis) were obtained from 119 patients. Small bars represent SD. Specific growth rate of primary tumours (PT-SGR) positively correlated with the expression of Ki67 (Jonckheere-Terpstra $\mathrm{p}=0.02$ ). 


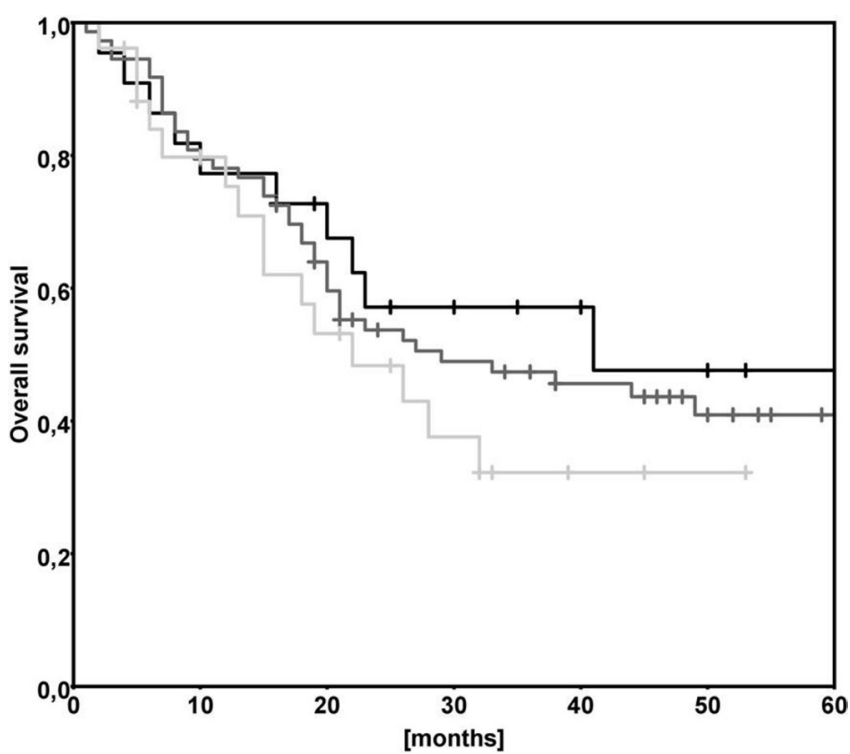

Figure 4 Kaplan-Meier plot of specific growth rates (SGRs) of primary tumours. SGRs of primary tumours grouped by low (black line; SGR $<0.3 \% /$ day; $n=22$ ), medium (dark grey line; $0.3 \% \leq S G R<3 \% / d a y ; n=73$ ) and high (pale grey line; SGR $>3 \% /$ day; $n=26$ ) growth rates. $X$-axis represents time in months, Y-axis overall survival. The survival curves of the three SGR groups did not differ significantly (log-rank $\mathrm{p}=0.45)$.

was approximately $16 \mathrm{~mL}$ (median $\approx 9 \mathrm{~mL}$ ). In planning CTs of the same patients 3-4 weeks later, mean PT-volume was approximately $25 \mathrm{~mL}$ (median $\approx 17 \mathrm{~mL}$; table 2 ). These PT-volumes are in line with previous publications reporting mean volumes of $11-37 \mathrm{~mL} \cdot{ }^{23-26}$ Mean LN-volume measured in diagnostic CTs was $9 \mathrm{~mL}$ (median $\approx 2 \mathrm{~mL}$ ) and approximately $15 \mathrm{~mL}$ (median $\approx 4 \mathrm{~mL}$ ) in planning CTs (table 2). Previously reported mean LN-volumes were considerably larger with $22-25 \mathrm{~m} .{ }^{27} 28$ However, in these studies, the volume of all pathological cervical lymph nodes was measured instead of only the largest one as in this study.

Tumour and pathological lymph node growth can be reported as DT or SGRs. DT is the number of days the tumour needs to double its volume. ${ }^{4}$ The lower the DT, the faster the tumour growth. Since DT was frequently reported in earlier studies, it was provided for comparison. SGR is defined as relative increase of volume in per cent per day. The resulting variable is constant, linear and independent of the initially measured volumes. ${ }^{5}$ SGR is considered less affected by measurement uncertainties of short time intervals and minor volume differences than DT. $^{5}$

For both DT and SGR, tumour volumes are required. A volume approximation method from CT scans, which reflects frequently available diagnostics, was used here. This approximation was based on measurements of maximum orthogonal diameters, similar to a formula described in 1990 by MacDonald and coworkers to approximate volumes of brain tumours. ${ }^{29}$ The method employed an ellipsoid formula, which was applicable over a wide range of $\mathrm{PT}$ and $\mathrm{LN}$ sizes and resulted only in a slight underestimation of $8 \%$ of the PT-volume measured with the reference method. ${ }^{15}$ A similar approximation method has also been used by Jensen and coauthors. ${ }^{30}$

More sophisticated segmentation and more modern imaging techniques may have allowed for better tumour margin delineation. However, the first require specific workstations with limited availability ${ }^{15}$ (ie, semiautomated or automated slice-by-slice segmentation) and large interobserver variations may remain. ${ }^{31}$ The latter may allow better visualisation of oropharyngeal and oral HNSCC (ie, MRI) or better visualisation of locally advanced tumours (ie, fluorodeoxyglucose PET; FDG-PET). However, both imaging techniques are not as frequently available as CT scans and other limitations apply. For MRIs, blurred tumour margins may be observed if patients swallow or breathe; for FDG-PET, a lack of spatial resolution may be disadvantageous in smaller HNSCC tumours. ${ }^{31}$

Mean PT-SGRs in incident HNSCC was $1.8 \% /$ day (table 2), unimodally distributed, slightly right skewed and independent of the initial PT-volume, which supports the basic concept underlying SGR calculation. The interval between diagnostic CT and planning CT did not influence PT-SGR $(p=0.1)$, suggesting that no marked growth deceleration occurred with longer waiting times. Interestingly, PT-SGRs did not significantly differ by tumour site $(\mathrm{p}=0.6$; table 3). Murphy and colleagues observed a lower median SGR of $0.74 \% /$ day in patients with oropharyngeal cancer. ${ }^{6}$ However, the authors did not include sites with faster tumour growth such as the oral cavity (median SGR 2.4\%/day) and hypopharynx (median SGR 2.0\%/day). Moreover, most of the patients reported by Murphy and colleagues had T1 or T2 HNSCC and were p16 positive.

The median PT-DT in this study was 43 days. The 25 th percentile was 24 and the 75 th percentile 85 days. Median LN-DT was 41 days. The 25th percentile was 25 and the 75 th percentile 80 days (table 2 ). Jensen and coauthors reported a slower growth rate in 61 patients with HNSCC with a median PT-DT of 99 days. The authors, however, additionally stated that half of the patients showed a faster growth rate with a PT-DT of 30 days. ${ }^{30}$ When compared with DT of other solid tumours, this means that HNSCC reveals rapid tumour growth. For breast cancer, Steel and Lamerton reported a median DT of 285 days. ${ }^{4}$ For lung cancer, DTs vary depending on histology between median 42 days for metastases as reported by Loeffler and colleagues and 181 for non-small cell lung cancer as reported by Winer-Muram and coworkers. ${ }^{42}$ For pancreatic adenocarcinoma, Furkawa and colleagues reported a mean PT-DT of 144 days. ${ }^{32}$ For sarcomas, median PT-DTs of 35 days were reported by Blomqvist and coauthors. ${ }^{32}$ Additional median PT-DTs for additional solid tumours are provided in table 4.

PT-SGRs significantly correlated with the expression of EGFR, Ki67 and CD44 ( $\mathrm{p}=0.021, \mathrm{p}=0.018$ and $\mathrm{p}=0.031$, respectively) with higher PT-SGRs in patients with higher expression of the three biomarkers (figure 3). These correlations appear biologically sound. A correlation 
Table 4 Reported tumour volume doubling times for selected solid tumours*

\begin{tabular}{lcr}
\hline Tumour & $\begin{array}{l}\text { Median DT } \\
\text { (days) }\end{array}$ & n \\
\hline Present study $^{30}$ & 47 & 123 \\
HNSCC $^{30}$ & $99 \dagger$ & 61 \\
\hline Breast cancer $^{4}$ & 285 & 16 \\
\hline Lung $_{\text {bronchioalveolar carcinoma }}^{32}$ & 181 & 9 \\
Non-small cell lung carcinoma $^{32}$ & 181 & \\
\hline Lung metastasis $^{4}$ & 42 & 6 \\
Pancreatic adenocarcinoma $^{32}$ & 144 & 24 \\
Sarcoma $^{32}$ & 35 & 9 \\
\hline
\end{tabular}

${ }^{*}$ Modified after Mehrara and coauthors. ${ }^{32}$

†Jensen and co-workers also stated tumour volume doubling times for the faster growing half of the patients with a median PTDT of $30 .^{30}$

of clinical tumour growth rates and expression of these biomarkers within the tumour of the same patients was not yet reported. EGFR is a cell surface receptor which promotes proliferation, invasion, angiogenesis and metastatic spread in HNSCC, if overexpressed. ${ }^{8} \mathrm{Ki}-67$ is a nuclear protein expressed on cells in all phases of the cell cycle except in G0 phase. Thus, its expression marks the total fraction of proliferating cells in a tumour. ${ }^{9}$ In laryngeal and hypopharyngeal SCC, an association between Ki67 expression and advanced tumour stages has been reported. ${ }^{10}{ }^{11}$ In line with our observation of a correlation of CD44 expression and PT-SGR, an association between advanced $\mathrm{T}$ categories and high CD44 expressions has been reported in a meta-analysis including 30 studies with 2102 patients. ${ }^{12}$ No significant correlation between PT-SGR and $\mathrm{p} 16$ expression was observed $(\mathrm{p}=0.81)$. This observation differs from previous observations made by Murphy and colleagues. The authors observed that p16 expression correlated well with PT-SGR with faster growth rates in p16-negative HNSCCs. However, the authors included oropharyngeal HNSCC only and the majority $(79 \%)$ were p16-positive tumours. ${ }^{6}$ When used as a covariate in a Cox model, PT-SGR had no significant effect on OS (log-rank $\mathrm{p}=0.054)$. No significant differences of survival curves were also observed if PT-SGRs were categorised in groups of low, medium and high growth rates $(\log$-rank $\mathrm{p}=0.45$; figure 4$)$. In contrast, van Bockel and coworkers observed a significant association between PT-SGR and OS in laryngeal cancer. ${ }^{7}$

To our knowledge, no data on lymph node specific growth rates (LN-SGR) in HNSCC have been previously reported. In the investigated patients, the mean LN-SGR was 1.7\%/day, similar to mean PT-SGR (table 2). In contrast to PT-SGR, LN-SGR did depend on initial LN-volumes in diagnostic CT scans $(\mathrm{p}=0.003)$ with higher LN-SGRs in lymph nodes with larger initial LN-volumes. Also, the interval between diagnostic CT and planning CT significantly influenced LN-SGR ( $\mathrm{p}=0.003$ ). The longer the interval, the lower the observed LN-SGR, suggesting growth slowdown. Moreover, LN-SGR was significantly influenced by tumour site $(\mathrm{p}=0.03$; table 3$)$, with smallest growth rates in lymph nodes from laryngeal HNSCC. In contrast to PT-SGR, LN-SGR did not depend on expressions of EGFR, Ki67, CD44 nor p16 expression in primary tumours $(\mathrm{p}=0.115, \mathrm{p}=0.311, \mathrm{p}=0.746$ and $\mathrm{p}=0.809$, respectively). In LN, SGRs had no significant impact on OS either $(\mathrm{p}>0.05)$.

New cartilage or bone infiltrations during the observation period were observed in 10 patients and new central lymph node necroses were observed in eight patients. SGRs had no significant impact on the development of either of them. Both initial cartilage or bone infiltration and central lymph node necrosis were associated with worse survival. However, probably due to the low number of events, no significant impact of new cartilage or bone infiltration nor new central lymph node necrosis on survival was observed in Kaplan-Meier analysis (both $\log$-rank $\mathrm{p}>0.05$ ).

Certain limitations of the present study need to be addressed. First, this small numbered retrospective study predominantly exploring patients with advanced-stage oropharyngeal HNSCC should be supplemented by a larger, prospective investigation, which also included patients with limited disease of all common HNSCG tumour sites. Second, the approximation $\operatorname{method}^{15}$ proposed here may be easily performed from frequently available CT scans over a wide range of different PT-volumes and LN-volumes. However, more sophisticated segmentation and more modern imaging techniques may have allowed for better tumour margin delineation and hence form more accurate SGRs. Third, the comparably short interval between diagnostic CT scans and planning CT scans may be considered positive from a clinical perspective. However, in terms of exploring SGRs in advanced-stage HNSCC, this interval may not be sufficient to accurately determine great changes in PT-volume and LN-volume.

\section{CONCLUSION}

HNSCCs are rapidly growing malignancies. Primary tumours and lymph nodes grow more than $1 \%$ per day. Consequently, time matters, and treatment must not be delayed.

Contributors DD has written major parts of the manuscript and performed the calculations of tumour and lymph node volumes. TS was involved in establishing the study concept, design and the internal review process of the manuscript. VHS established the population-based tumour registry from which patients were enrolled. He further obtained the ethics committee approval for the study. NF performed additional tumour and pathological cervical lymph node measurements and was involved in the internal review process of the manuscript. MA has performed additional calculations and database work in the population-based tumour registry. JD performed the immunohistochemical staining of the tumour biopsies and was involved in establishing the study concept, design and the internal review process of the manuscript. AP provided the radiotherapy-planning CT scans and performed performed additional tumour and pathological cervical lymph node 
measurements. GW supervised the tumour and pathological cervical lymph node measurements as an experienced head and neck radiologist. HR supervised the writing process of the manuscript and performed all statistical calculations in the manuscript.

Funding The authors have not declared a specific grant for this research from any funding agency in the public, commercial or not-for-profit sectors.

Competing interests None declared.

Patient consent for publication Obtained.

Ethics approval The review board of the Medical University of Innsbruck had approved the study (UN4590). All procedures performed in studies involving human participants were in accordance with the ethical standards of the institutional review board and with the 1964 Helsinki declaration and its later amendments or comparable ethical standards.

Provenance and peer review Not commissioned; externally peer reviewed. Data sharing statement № additional data from the study are available. Open access This is an open access article distributed in accordance with the Creative Commons Attribution Non Commercial (CC BY-NC 4.0) license, which permits others to distribute, remix, adapt, build upon this work non-commercially, and license their derivative works on different terms, provided the original work is properly cited, appropriate credit is given, any changes made indicated, and the use is non-commercial. See: http://creativecommons.org/licenses/by-nc/4.0/.

\section{REFERENCES}

1. Murphy CT, Galloway TJ, Handorf EA, et al. Increasing time to treatment initiation for head and neck cancer: an analysis of the National Cancer Database. Cancer 2015;121:1204-13.

2. Murphy CT, Galloway TJ, Handorf EA, et al. Survival impact of increasing time to treatment initiation for patients with head and neck cancer in the United States. J Clin Oncol 2016;34:169-78.

3. Stefanuto P, Doucet JC, Robertson C. Delays in treatment of oral cancer: a review of the current literature. Oral Surg Oral Med Oral Pathol Oral Radiol 2014;117:424-9.

4. Steel GG, Lamerton LF. The growth rate of human tumours. $\mathrm{Br} J$ Cancer 1966;20:74-86.

5. Mehrara E, Forssell-Aronsson E, AhIman H, et al. Specific growth rate versus doubling time for quantitative characterization of tumor growth rate. Cancer Res 2007;67:3970-5.

6. Murphy CT, Devarajan K, Wang LS, et al. Pre-treatment tumorspecific growth rate as a temporal biomarker that predicts treatment failure and improves risk stratification for oropharyngeal cancer. Oral Oncol 2015;51:1034-40.

7. van Bockel LW, Verduijn GM, Monninkhof EM, et al. The importance of actual tumor growth rate on disease free survival and overall survival in laryngeal squamous cell carcinoma. Radiother Oncol 2014;112:119-24.

8. Bossi P, Resteghini C, Paielli N, et al. Prognostic and predictive value of EGFR in head and neck squamous cell carcinoma. Oncotarget 2016;7:74362-79.

9. Scholzen T, Gerdes J. The Ki-67 protein: from the known and the unknown. J Cell Physiol 2000;182:311-22.

10. Ashraf MJ, Maghbul M, Azarpira N, et al. Expression of Ki67 and P53 in primary squamous cell carcinoma of the larynx. Indian $\mathrm{J}$ Pathol Microbiol 2010;53:661-5.

11. Wang JX, Zhang YY, Yu XM, Xm Y, et al. Role of centromere protein $\mathrm{H}$ and Ki67 in relapse-free survival of patients after primary surgery for hypopharyngeal cancer. Asian Pac J Cancer Prev 2012;13:821-5.

12. Chen J, Zhou J, Lu J, et al. Significance of CD44 expression in head and neck cancer: a systemic review and meta-analysis. BMC Cancer 2014;14:15.
13. Sobin LWC. TNM classification of malignant tumours: WileyBlackwell, 2009.

14. van den Brekel MW, Stel HV, Castelijns JA, et al. Cervical lymph node metastasis: assessment of radiologic criteria. Radiology 1990;177:379-84.

15. Dejaco D, Url C, Schartinger VH, et al. Approximation of head and neck cancer volumes in contrast enhanced CT. Cancer Imaging $2015 ; 15: 16$

16. Schwartz M. A biomathematical approach to clinical tumor growth. Cancer 1961;14:1272-94.

17. Dudás J, Schartinger VH, Romani A, et al. Cell cycle association and hypoxia regulation of excision repair cross complementation group 1 protein (ERCC1) in tumor cells of head and neck cancer. Tumour Biol 2014;35:7807-19.

18. Schartinger VH, Falkeis $\mathrm{C}$, Laimer $\mathrm{K}$, et al. Neuroendocrine differentiation in head and neck squamous cell carcinoma. $J$ Laryngol Otol 2012;126:1261-70.

19. Rietbergen MM, Snijders PJ, Beekzada D, et al. Molecular characterization of p16-immunopositive but HPV DNA-negative oropharyngeal carcinomas. Int J Cancer 2014;134:2366-72.

20. Smilek P, Dusek L, Veselý K, et al. Correlation of expression of Ki-67, EGFR, c-erbB-2, MMP-9, p53, bcl-2, CD34 and cell cycle analysis with survival in head and neck squamous cell cancer. J Exp Clin Cancer Res 2006;25:549-55.

21. Reimers N, Kasper HU, Weissenborn SJ, et al. Combined analysis of HPV-DNA, p16 and EGFR expression to predict prognosis in oropharyngeal cancer. Int J Cancer 2007;120:1731-8.

22. Schemper M, Smith TL. A note on quantifying follow-up in studies of failure time. Control Clin Trials 1996;17:343-6.

23. Dejaco D, Steinbichler T, Schartinger VH, et al. Prognostic value of tumor volume in patients with head and neck squamous cell carcinoma treated with primary surgery. Head Neck 2018;40:728-39.

24. Davis KS, Lim CM, Clump DA, et al. Tumor volume as a predictor of survival in human papillomavirus-positive oropharyngeal cancer. Head Neck 2016;38(Suppl 1):E1613-7.

25. Knegjens JL, Hauptmann M, Pameijer FA, et al. Tumor volume as prognostic factor in chemoradiation for advanced head and neck cancer. Head Neck 2011;33:375-82.

26. Oemus D, Inhestern J, Schmalenberg $\mathrm{H}$, et al. Prognostic value of tumor volumetry data of routine imaging data in a head and neck cancer registry. Eur Arch Otorhinolaryngol 2014;271:2531-7.

27. Chen SW, Yang SN, Liang JA, et al. Prognostic impact of tumor volume in patients with stage III-IVA hypopharyngeal cancer without bulky lymph nodes treated with definitive concurrent chemoradiotherapy. Head Neck 2009;31:709-16.

28. Doweck I, Denys D, Robbins KT. Tumor volume predicts outcome for advanced head and neck cancer treated with targeted chemoradiotherapy. Laryngoscope 2002:112:1742-9.

29. Macdonald DR, Cascino TL, Schold SC, et al. Response criteria for phase II studies of supratentorial malignant glioma. J Clin Oncol 1990;8:1277-80.

30. Jensen AR, Nellemann HM, Overgaard J. Tumor progression in waiting time for radiotherapy in head and neck cancer. Radiother Oncol 2007;84:5-10.

31. Grégoire V, Evans M, Le QT, et al. Delineation of the primary tumour Clinical Target Volumes (CTV-P) in laryngeal, hypopharyngeal, oropharyngeal and oral cavity squamous cell carcinoma: AIRO, CACA, DAHANCA, EORTC, GEORCC, GORTEC, HKNPCSG, HNCIG, IAG-KHT, LPRHHT, NCIC CTG, NCRI, NRG Oncology, PHNS, SBRT, SOMERA, SRO, SSHNO, TROG consensus guidelines. Radiother Oncol 2018;126:3-24.

32. Mehrara E, Forssell-Aronsson E, Ahlman $\mathrm{H}$, et al. Quantitative analysis of tumor growth rate and changes in tumor marker level: specific growth rate versus doubling time. Acta Oncol 2009;48:591-7. 